\title{
Effets combinés de l'apport d'acide caproïque et de la concentration en protéines sur l'utilisation de laits artificiels par l'agneau préruminant
}

\section{II. - Croissance et composition corporelle des agneaux}

\author{
B. AUROUSSEAU \\ I.N.R.A., Laboratoire d'Etude du Métabolisme énergétique \\ Centre de Recherches zootechniques et vétérinaires
}

F 63122 Ceyrat

Avec la collaboration technique de F. Duboisset, J.P. Donnat, S. Gasnet, G. Moins et R. Souchet

\begin{abstract}
Résumé
Les effets de la nature des lipides (suif-coprah, "SC » ou suif-coprah-tricaproine, «SCT»), de la teneur en protéines (moyenne, «M», 24 p. 100 de la matière sèche, MS, ou haute "H», 31 p. 100 de MS) ou de la quantité de tricaproïne utilisée $(4,5$ ou 11,4 p. 100 de MS) sur la vitesse de croissance, l'efficacité d'utilisation des aliments, et la composition corporelle de l'agneau mâle préruminant ont été étudiés au cours de 4 essais. Les études de croissance et d'efficacité alimentaire ont été effectuées entre les âges de 3 et de 21 jours sur 56 agneaux Limousins (essais 1, 2 et 4 ) et sur 16 agneaux Ile-de-France (essai 3). Les études de la composition corporelle ont été effectuées sur 24 agneaux Limousins abattus à l'âge de 3 semaines (essai 2).

Chez les agneaux Limousins, le gain de poids vif journalier moyen passe de $206 \mathrm{~g} / \mathrm{j}$ dans le cas des lots « SCM » à $263 \mathrm{~g} / \mathrm{j}$ dans le cas des lots «SCT-H» $(\mathrm{P}<0,05)$; il est intermédiaire dans le cas des lots « $\mathbf{S C H}$ » et «SCT-M». Chez les agneaux Ile-de-France, il est également accru de 14 p. 100 (NS) dans le cas des agneaux Ile-de-France qui reçoivent les laits «SCT ». L'efficacité alimentaire n'est pas modifiée par l'augmentation de la teneur du lait en protéines, mais est significativement accrue $(\mathrm{P}<0,05)$ chez les agneaux qui reçoivent de l'acide caprö̈que. Elle passe de $939 \mathrm{~g}$ de gain/g de MS ingérée et de 40,6 g gain/MJ d'énergie brute en moyenne dans le cas des groupes « $\mathrm{SC}$ » à 1084 ou à 48,8 respectivement dans le cas des groupes «SCT».

Les proportions pondérales du tractus digestif et de l'ensemble peau tête et pattes sont augmentées dans le cas des laits les plus riches en protéines, mais celles des autres tissus ne sont significativement modifiées ni par la nature des matières grasses ni par la teneur en protéines des laits. Les coefficients d'allométrie du développement du cinquième quartier de la carcasse, des os, des muscles et des tissus adipeux $(0,801 ; 1,275 ; 1,232$; 1,144 et 1,452 respectivement) également indépendants des traitements expérimentaux, sont cependant supérieurs, dans le cas des os et des muscles, aux valeurs habituelles en raison, semble-t-il, de la supplémentation des laits en méthionine et en lysine.

La composition chimique du corps vide des agneaux ou des tissus déposés et en particulier leurs teneurs élevées en protéines (189 $\mathrm{g} / \mathrm{kg}$ de poids vif vide ou $206 \mathrm{~g} / \mathrm{kg}$ de gain, en moyenne) confirme les effets de la supplémentation en acides aminés sur l'accrétion de protéines. De plus, la présence d'acide caprö̈que dans les laits conduit à l'obtention de coefficients d'allométrie plus élevés pour la fixation d'eau dans le corps des
\end{abstract}


agneaux et moins élevés pour la fixation de matière organique non protéique. En conséquence, la teneur en eau du corps des agneaux des lots "SCT» est accrue aux dépens de la teneur en matière organique non protéique.

Mots clés : acide caprö̈que, croissance, composition corporelle, agneau préruminant.

\section{Introduction}

Les quantités d'énergie ingérées par l'agneau préruminant sont un facteur primordial de l'expression de ses potentialités de croissance (WELch, VANDER NoOT \& Gilbreath, 1963 ; Chiou \& Jordan, 1973 ; Black \& Griffiths, 1975). Contrairement aux acides gras longs qui se déposent directement en quantités imporlantes dans les tissus du jeune agneau (NORTON, JAGUSCH \& Walker, 1970; Stokes \& WALKER, 1970), les acides gras à chaîne moyenne se déposent peu dans les tissus (Aurousseau, Theriez \& DANIEl, 1973). Leur catabolisme peut augmenter la production de liaisons riches en énergie et ainsi favoriser la fixation de protéines dans les tissus (Aurousseau, Vermorel \& Bouvier, 1983, chez le veau). Il était intéressant de préciser les effets de ce type de composés et de les comparer à d'autres facteurs de l'augmentation de l'accrétion protéique et de la croissance des animaux : augmentation de la teneur des laits de remplacement en matières azotées (WALKER \& FaichneY, 1964 ; Norton, JAgusch \& Walker, 1970) ou supplémentation en acides aminés indispensables (Walker \& KIrK, 1975; Dove, Pearce \& Tribe, 1977).

Un premier compte rendu des effets combinés de l'ingestion d'acide caproïque, de la teneur des laits en protéines et de l'âge sur l'utilisation digestive de l'aliment d'allaitement chez l'agneau préruminant a été publié antérieurement (Aurousseau, Vermorel \& Theriez, 1983). Nous présentons ici les résultats concernant l'efficacité de l'utilisation des divers aliments pour la croissance et les effets de l'ingestion d'acide caproïque sur la composition corporelle du jeune agneau.

\section{Matériel et méthodes}

\section{A. Schéma expérimental}

Huit types d'aliments d'allaitement combinant 2 niveaux de concentration azotée (moyen « $\mathrm{M}$ » et haut « $\mathrm{H}$ », comportant respectivement 24 ou 30 p. 100 de matières azotées, par rapport à la MS, 2 types de matières grasses (mélange suif-coprah "SC », ou mélange suif-coprah-tricaproïne, «SCT ») et 2 taux d'incorporation $(4,5$ ou 11,3 p. 100 de MS) de tricaproïne (TC6) ont été utilisés au cours de 4 essais.

Chacun des essais 1,2 et 4 a mis en jeu 4 groupes d'agneaux mâles Limousins (4 agneaux par groupe pour les essais 1 et $4 ; 6$ agneaux par groupe pour l'essai 2) et l'essai 3 a mis en jeu 4 groupes de 4 agneaux mâles Ile-de-France. Les agneaux étaient répartis en lots homologues et recevaient les régimes expérimentaux à l'âge de 4 jours pour les essais 1,3 et 4 , ou dès la naissance pour l'essai 2 . 


\section{'TABLeaU 1}

Composition du lait ingéré par les animaux des différents groupes.

Composition of the milk ingested by the lambs of different groups.

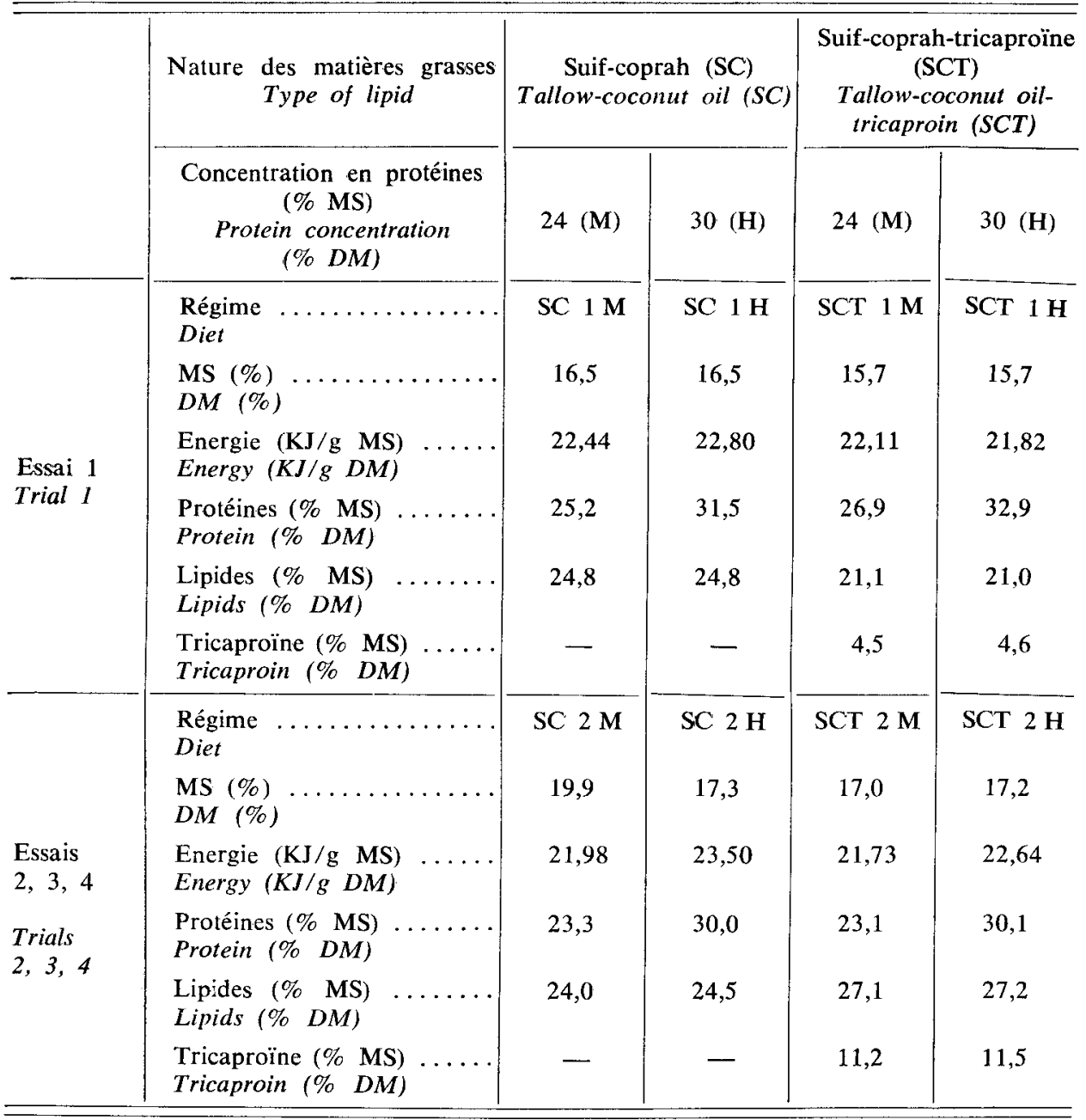

Le détail de la constitution des aliments a été décrit dans une précédente communication (Aurousseau, Vermorel \& Theriez, 1983) et 1a composition de l'ingéré pour chaque type de lait et pour chaque essai est rappelée dans le tableau 1.

Les animaux ont été maintenus au stade préruminant jusqu'à la fin de la cinquième semaine pour les essais 1 et 3 ou à la fin de la troisième semaine pour les essais 2 et 4 . Ils ont été abattus après 3 semaines d'expérience dans le cas de l'essai 2. 
Au cours du premier essai, les animaux, maintenus en cage individuelle en deuxième puis en cinquième semaine pour les mesures de digestibilité, ont été nourris en groupe au sol entre ces 2 périodes. Lors des 3 autres essais, ils ont été maintenus en cage individuelle pendant toute la durée des mesures. Dans les essais 2 et 3, les animaux étaient maintenus à l'attache dans des cages dont le plancher constitué d'un grillage était placé $80 \mathrm{~cm}$ au-dessus du sol et pour l'essai 4 ils étaient libres dans des cases individuelles dont le plancher situé au niveau du sol, était constitué d'un caillebottis en bois.

Les quantités d'aliments d'allaitement distribuées ou refusées ont été contrôlées individuellement sauf entre la deuxième et la cinquième semaines dans le cas de l'essai 1 (contrôle global pour chaque lot). La composition du lait réellement ingéré a été déterminée chaque semaine pour les 4 types d'aliments et au cours des 4 essais en procédant pour chaque période à l'analyse du lait distribué ou refusé par l'ensemble des animaux de chaque groupe selon les méthodes décrites antérieurement (Aurousseau, Vermorel \& Theriez, 1983).

Les animaux ont été pesés à la naissance puis chaque semaine, en début et en fin de période de mesures de digestibilité en particulier.

A la fin du deuxième essai, les animaux ont été abattus et leurs carcasses disséquées. Pour chaque animal, on a contrôlé lors de l'abattage le poids du contenu du tube digestif et relevé les poids du sang recueilli, du foie, de l'ensemble du tube digestif, du cœur et des poumons et celui de la peau additionnée de la tête et des pattes (l'ensemble de ces tissus correspond au cinquième quartier).

Les carcasses stockées pendant une période de 24 heures à $+4^{\circ} \mathrm{C}$ ont ensuite été disséquées en muscles totaux, os de la carcasse, tissus adipeux sous-cutanés, internes et intermusculaires. Les différentes données obtenues ont été corrigées pour les pertes de poids lors du stockage initial à $+4{ }^{\circ} \mathrm{C}$ pendant 24 heures.

Pour chaque agneau, l'ensemble des tissus de la carcasse d'une part ou du cinquième quartier d'autre part, ont été regroupés et soumis à un premier broyage dans l'azote liquide à l'aide d'un broyeur à hélice. Pour parfaire l'homogénéisation, $700 \mathrm{~g}$ environ du broyat obtenu sous forme de poudre congelée ont été soumis à un second broyage à l'aide d'un broyeur à lame de type Robotcoupe et des échantillons représentatifs de $120 \mathrm{~g}$ du produit obtenu prélevés et analysés aussitôt.

Les teneurs en énergie et en azote des échantillons ont été déterminées sur les produits lyophilisés respectivement à l'aide d'un calorimètre adiabatique Gallenkamp ou selon la méthode Kjeldahl. Elles ont été corrigées en fonction de leur teneur en matière sèche obtenue en parallèle par dessication à l'étuve $\left(24\right.$ heures à $\left.100^{\circ} \mathrm{C}\right)$. Leur teneur en cendres était obtenue par incinération au four à $700^{\circ} \mathrm{C}$ pendant 8 heures.

Les teneurs en protéines ont été calculées en affectant le coefficient 6,25 aux teneurs en azote et les teneurs en matière organique non protéique par différence entre la teneur en matière sèche et celles en protéines et en cendres.

Les résultats individuels d'efficacité de l'utilisation de la matière sèche et de l'énergie ingérée pour la croissance ont été soumises à une analyse de variance, selon une classification à 3 voies (effets de la nature des matières grasses du lait, de la concentration azotée et de la répétition) avec des effectifs différents (SNEDECoR \& CochraN, 1971). 


\section{Résultats}

\section{A. Appétibilité des régimes}

Dans le cas du premier essai, les agneaux des 4 lots présentent des consommations de lait identiques jusqu'au troisième jour, mais, par la suite, ceux qui reçoivent le lait $\mathrm{SCT} 1 \mathrm{H}$ montrent des consommations significativement plus élevées que les 3 autres lots $(+25$ p. 100, $\mathrm{P}<0,05$; fig. 1$)$. Le phénomène n'est cependant pas retrouvé au cours des essais suivants (tabl. 2), ni avec les animaux Limousins (essais 2 et 4 ), ni avec les animaux Ile-de-France (essai 3).

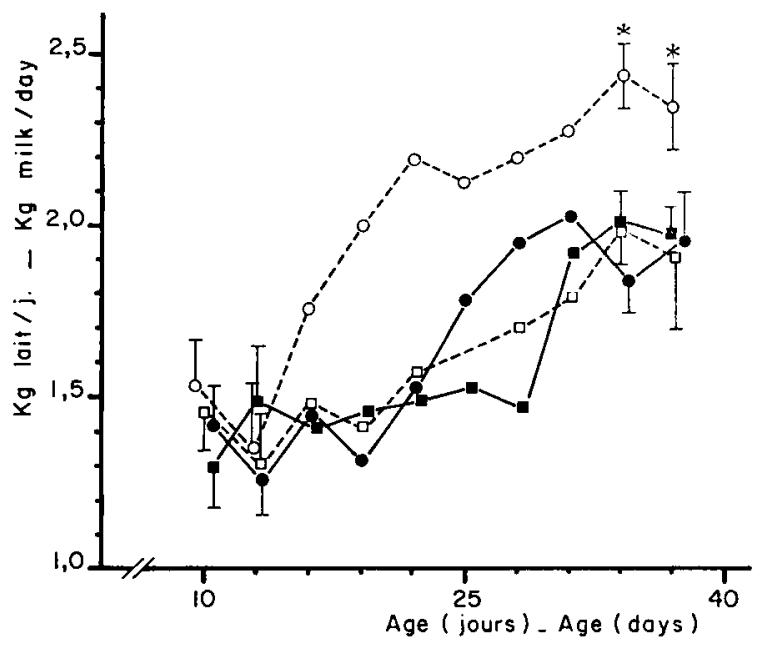

FIG. 1

Effet de l'adjonction d'acide caproüque dans les laits sur leur appétibilité dans l'essai 1. Les valeurs individuelles de consommations (4 agneaux par lot) ont été contrôlés seulement en deuxième et en cinquième semaines.

Effect of adding caproic acid to the milk on its palatability.

Individual levels of intake (4 lambs in each group) were recorded only during the second and fifth weeks.

alait «SC 1M»; • lait "SC $1 \mathrm{H} » ; \square$ lait «SCT 1M ; O lait «SCT $1 \mathrm{H}$ ».

- $S C$ IM» milk; - «SC $1 H$ » milk; $\square$ «SCT $1 M$ » milk; O «SCT $1 H$ » milk.

* valeurs significativement supérieures à leurs homologues $(\mathrm{P}<0,05)$.

* data significantly higher than their counterparts $(P<0.05)$.

\section{B. Croissance des agneaux}

Dans le cas des agneaux Limousins et pour le premier essai, les gains de poids quotidiens des animaux qui reçoivent les laits SC $1 \mathrm{M}, \mathrm{SC} 1 \mathrm{H}$ et $\mathrm{SCT} 1 \mathrm{M}$ sont identiques $(225 \mathrm{~g} / \mathrm{j}$ environ) et inférieurs de $25 \mathrm{p} .100(\mathrm{P}<0,05)$ à celles des 


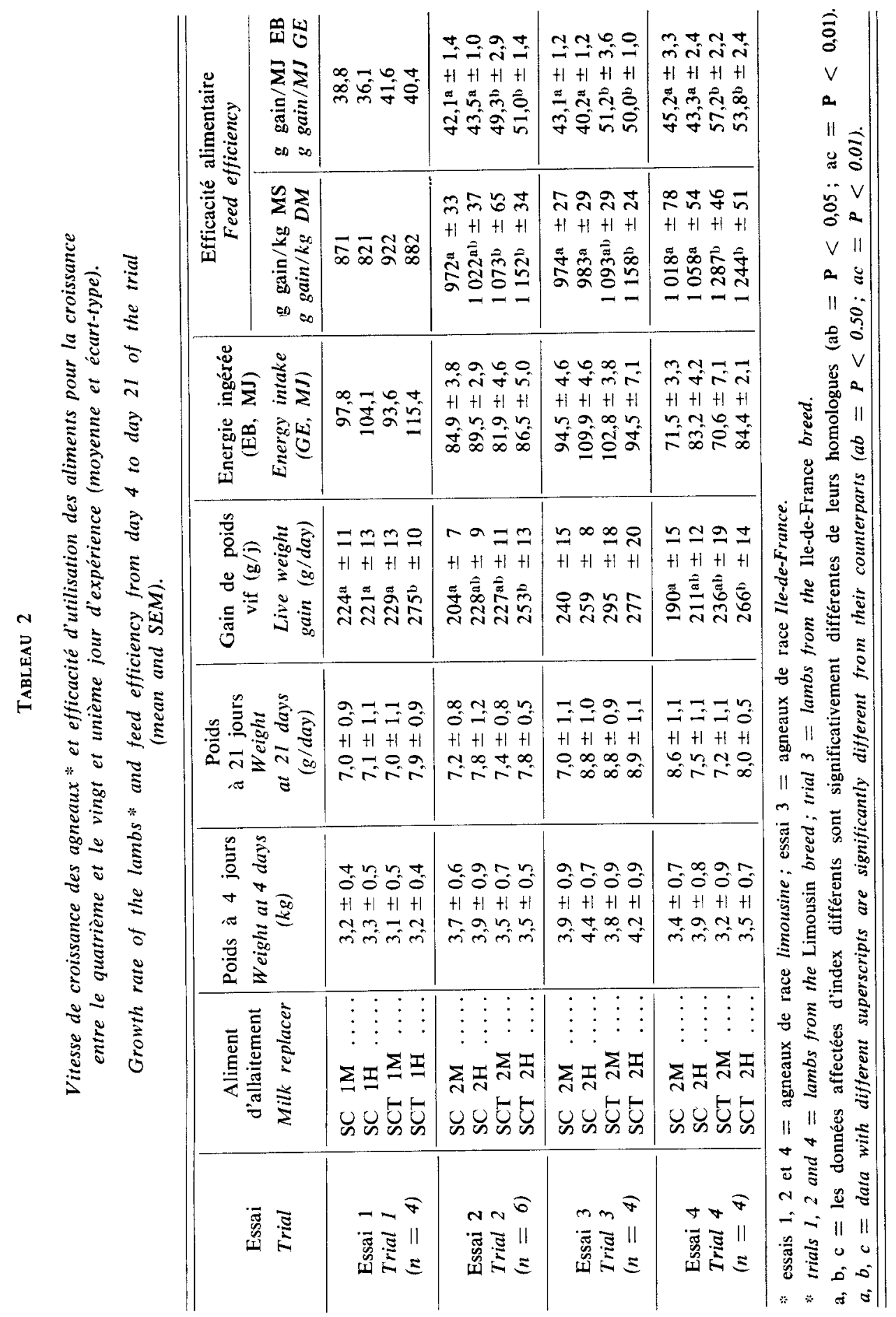


animaux qui reçoivent l'aliment SCT $1 \mathrm{H}$ (tabl. 2). Au cours du second et du quatrième essais, les vitesses de croissance les plus faibles $(200 \mathrm{~g} / \mathrm{j}$ environ $)$ sont obtenues avec l'aliment d'allaitement SC $2 \mathrm{M}$. Elles sont plus élevées ( $225 \mathrm{~g} / \mathrm{j}$ environ) avec les aliments SC $2 \mathrm{H}$ et SCT $2 \mathrm{M}$ (NS) et enfin les plus élevées ( $260 \mathrm{~g} / \mathrm{j}$ environ) sont obtenues avec le lait SCT $2 \mathrm{H}$ : l'accroissement par rapport aux valeurs obtenues avec le lait SC $2 \mathrm{M}(+30$ p. 100) est significatif $(\mathrm{P}<0,05)$.

Dans le cas des agneaux Ile-de-France, les vitesses de croissance les plus élevées sont obtenues avec les laits comportant de la tricaproïne (augmentation de 14 p. 100 en moyenne dans le cas des lots SCT $2 \mathrm{M}$ et SCT $2 \mathrm{H}$ comparés aux lots SC $2 \mathrm{M}$ et $\mathrm{SC} 2 \mathrm{H})$. Cependant, le petit nombre d'animaux employé et l'importante variabilité des différents paramètres ne permettent pas de comparer avec précision les performances des différents lots.

\section{Efficacité d'utilisation des aliments pour la croissance}

Compte tenu de délais variables entre la naissance des agneaux et leur installation dans les cages ainsi que des contraintes liées à l'abattage des agneaux utilisés lors du deuxième essai, les données complètes pour l'ensemble des animaux utilisés au cours des 4 essais ne sont disponibles qu'entre les âges de 4 et 21 jours. Cette période a donc été choisie pour comparer l'efficacité d'utilisation des différents aliments pour la croissance.

Au cours de l'essai 1, l'augmentation de la teneur des laits en matières azotées diminue de 5,0 ou de 4,9 p. 100 en moyenne les efficacités alimentaires respectives de la MS ( $g$ gain/kg MS) ou de l'énergie (g gain/MJ EB), quel que soit le type de matières grasses employées. En revanche l'adjonction d'acide caproïque dans les laits, quelle que soit leur teneur en matières azotées, améliore ces paramètres de 6,6 ou de 9,7 p. 100 en moyenne (tabl. 2). L'absence de données individuelles (consommations) ne permet pas d'apprécier la signification statistique de ces différences.

Pour les autres essais, l'analyse de variance (tabl. 3) montre que l'augmentation de la concentration azotée du lait améliore significativement $(P<0,05)$ l'efficacité d'utilisation de la matière sèche pour la croissance. Cependant, à l'intérieur de chacun des essais, aucune des différences entre lots homologues n'atteint le seuil de signification (tabl. 2). De plus, en raison des différences de teneur en énergie de chacun des aliments, les écarts d'efficacité d'utilisation de l'énergie pour la croissance sont plus faibles (tabl. 1).

Par ailleurs, l'effet du remplacement d'une partie des matières grasses par de la tricaproïne explique à lui seul respectivement 38 ou 45 p. 100 de la variabilité totale de l'efficacité d'utilisation de la matière sèche ou de l'énergie pour la croissance de l'agneau, ce qui correspond à une amélioration hautement significative $(\mathrm{P}<0,005)$ de ces paramètres. Ainsi, les efficacités alimentaires respectives de la MS et de l'énergie sont améliorées en moyenne, quelle que soit la concentration en matières azotées du lait, de 14,2 et de 15,6 p. 100 au cours de l'essai 2, ou de 22,1 et de 25,3 p. 100 au cours de l'essai $4(\mathrm{P}<0,01)$ dans le cas des agneaux Limousins. Elles sont améliorées de 15,0 et de 21,6 p. 100 au cours de l'essai 3 $(\mathrm{P}<0,01)$, avec les agneaux Ile-de-France. 


\section{TABleaU 3}

Influence combinée de la nature des matières grasses utilisées, de la concentration azotée des laits et de l'essai considéré sur l'efficacité d'utilisation pour la croissance de l'agneau de la matière sèche et de l'énergie.

Combined effects of type of lipid, milk protein content and trial replication on dry matter and energy efficiency in growing lambs.

\begin{tabular}{l|c|c|c|c|c}
\hline \hline & \multicolumn{1}{c|}{$\begin{array}{c}\text { Degrés } \\
\text { Paramètres (1) } \\
\text { Parameters (1) }\end{array}$} & $\begin{array}{c}\text { Efficacité de la } \\
\text { matière sèche } \\
\text { Efficiency of dry matter }\end{array}$ & $\begin{array}{c}\text { Efficacité de l'énergie } \\
\text { Efficiency of energy }\end{array}$ \\
\cline { 2 - 5 } & $\begin{array}{c}\text { Degrees } \\
\text { of freedom }\end{array}$ & $\begin{array}{c}\text { S.C.E (2) } \\
\text { S.S. (2) }\end{array}$ & $\begin{array}{c}\text { Niveau de } \\
\text { probabilité } \\
\text { Probability } \\
\text { level }\end{array}$ & $\begin{array}{c}\text { S.C.E (2) } \\
\text { S.S. (2) }\end{array}$ & $\begin{array}{c}\text { Niveau de } \\
\text { probabilité } \\
\text { Probability } \\
\text { level }\end{array}$ \\
\hline $\begin{array}{l}\text { Erreur ........... } \\
\text { Error }\end{array}$ & 47 & 0,5095 & - & 18484,6 & - \\
$\begin{array}{l}\text { Concentration azotée } \\
\text { Protein level }\end{array}$ & 1 & 0,0414 & $\mathrm{P}<0,05$ & 5,9 & N.S. \\
$\begin{array}{l}\text { Nature des lipides .. } \\
\text { Type of lipids }\end{array}$ & 1 & 0,4069 & $\mathrm{P}<0,005$ & 18809,9 & $\mathrm{P}<0,005$ \\
$\begin{array}{l}\text { Répétition .......... } \\
\text { Replication }\end{array}$ & 2 & 0,1159 & $\mathrm{P}<0.01$ & 3354,6 & $\mathrm{P}<0,025$ \\
\hline $\begin{array}{l}\text { (1) aucune interaction - no interaction. } \\
\text { (2) sommes des carrés des écarts - sum of deviation squares. }\end{array}$ &
\end{tabular}

Enfin, 10 p. 100 de la variabilité totale de l'efficacité d'utilisation des aliments pour la croissance est due aux différences observées d'un essai à l'autre ce qui correspond à un effet significatif $(P<0,025)$ de l'essai considéré, une plus grande efficacité d'utilisation des laits étant observée dans le quatrième essai (tabl. 2).

\section{Composition corporelle des agneaux}

Les poids du sang recueilli (3,7 p. 100 du poids vif), du foie ( 3 p. $100 \mathrm{du}$ poids vif) ou du cœur et des poumons ( 7 p. $100 \mathrm{du}$ poids vif) sont indépendants des traitements expérimentaux. En revanche, les poids du tube digestif ou de l'ensemble peau, tête et pattes $(6,6$ p. 100 et 19,2 p. 100 du poids vif respectivement, dans le cas des aliments contenant 24 p. 100 de matières azotées), sont accrus respectivement de 19,2 et de 13,6 p. $100(P<0,05)$ lorsque la teneur en matières azotées des laits est augmentée, quelle que soit la nature des matières grasses (annexe 1).

Les poids des autres parties du corps des animaux ne sont influencées par aucun des traitements. Le rendement en carcasse (rapport entre le poids de carcasse et le poids du corps vide des agneaux) s'élève en moyenne, à 57 p. 100 pour les 


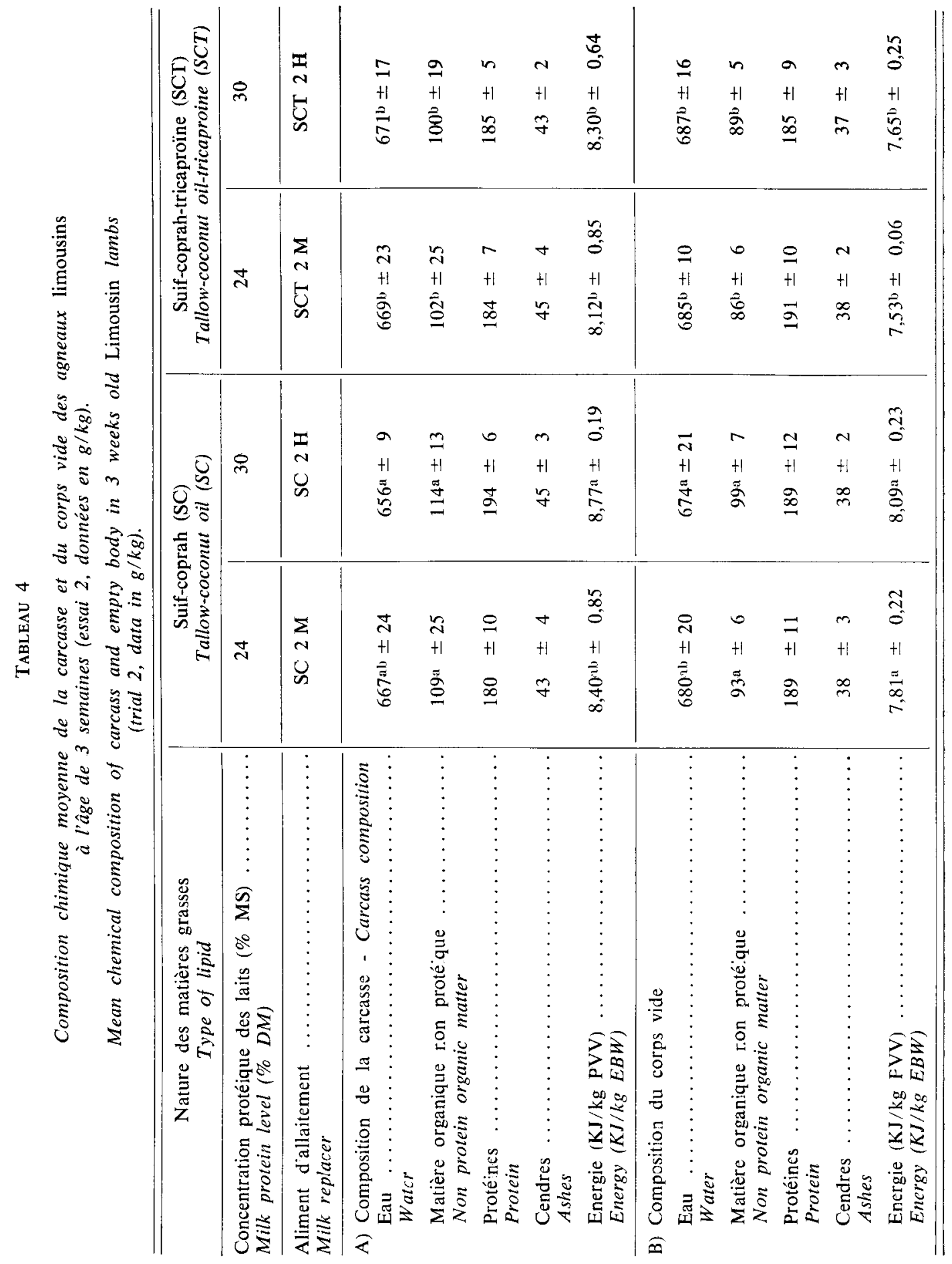


animaux des 4 lots, tandis que les os, les muscles et les tissus adipeux représentent respectivement $23,1,61,9$ et 14,9 p. 100 environ du poids de la carcasse. Enfin, les tissus adipeux internes, sous-cutanés ou intermusculaires représentent, en moyenne, respectivement $22,9,15,6$ ou 61,5 p. 100 du poids des tissus adipeux totaux.

L'estimation de la composition de la carcasse des agneaux à la naissance d'après les données de BroAD \& Davies (1980 b) permet de calculer que le développement du cinquième quartier, de la carcasse, des muscles, des os et des tissus adipeux totaux admettent relativement au poids vif vide des animaux des coefficients d'allométrie de $0,801 \pm 0,110,1,275 \pm 0,065,1,232 \pm 0,084,1,144 \pm 0,117$ et $1,452 \pm 0,163$. Ces coefficients sont indépendants à la fois de la nature des matières grasses utilisées et de la concentration azotée des laits.

\section{E. Composition chimique des agneaux}

L'augmentation de la teneur en protéines de l'aliment, quel que soit le type de matières grasses utilisées, ne modifie pas la composition chimique de la carcasse ou celle du corps vide des agneaux (tabl. 4). Par contre, l'ingestion d'acide caproïque, quelle que soit la teneur en protéines de l'aliment, augmente la teneur en eau des carcasses et du corps vide des agneaux (de 2 p. 100 environ, $P<0,05$ dans le cas d'aliments riches en matières azotées). La présence de cet acide dans les laits de remplacement provoque, par ailleurs, une diminution significative $(P<0,05)$ de la teneur des carcasses en matière organique non protéique $(-14,0$ et $6,9 \mathrm{p}$. 100 respectivement pour les laits à teneur élevée ou à teneur normale en protéines) ou en énergie $(-5,6$ et $-3,4$ p. 100 respectivement) ainsi que de la teneur du corps vide en matière organique non protéique $(-11,2$ et $-8,1$ p. 100 respectivement) ou en énergie $(-5,8$ et $-3,7$ p. 100 respectivement). Les teneurs en protéines et en matières minérales restent cependant inchangées.

En estimant la composition chimique des carcasses ou du corps vide des agneaux à la naissance d'après les données obtenues au laboratoire et partiellement publiées (Villette \& Aurousseau, 1981), il a été possible de calculer les coefficients d'allométrie de fixation des différents constituants dans la carcasse ou le corps entier des animaux (tabl. 5) et la composition des tissus déposés. La teneur en protéines des aliments d'allaitement ne modifie pas les vitesses relatives d'accumulation des différents constituants chimiques dans la carcasse des animaux ou dans leur corps entier. En revanche, l'ingestion d'acide caproïque accroît $(P<0,01)$ les vitesses relatives de fixation d'eau dans la carcasse $(1,180$ contre 1,141$)$ ou le corps entier $(0,852$ contre 0,826$)$ des agneaux; ainsi, la présence d'acide caprö̈que dans les aliments augmente $(P<0,01)$ la teneur en eau des tissus déposés dans la carcasse ou dans le corps entier des agneaux de 6,4 ou de 4,0 p. 100 respectivement dans le cas des laits à teneur moyenne en protéines et de 1,3 ou de 2,3 p. 100 dans le cas des laits à teneur élevée en protéines. En contrepartie, cet acide ralentit $(\mathrm{P}<0,05)$ les vitesses relatives de fixation de matière organique non protéique ou d'énergie dans la carcasse (2,011 contre 2,232 et 1,861 contre 2,019 respectivement) ou dans le corps entier des agneaux (2,253 contre 2,545 et 1,615 contre 1,773 respectivement). Il réduit de façon significative $(P<0,01)$ les teneurs en matière organique non protéique et en énergie des tissus déposés dans les carcasses (-22,4 et $-12,3$ p. 100 respectivement dans le cas des laits à teneur moyenne en protéines, « $\mathrm{M} » ;-11,6$ et $-6,3$ p. 100 respectivement dans le cas des laits à teneur élevée en protéines, « $\mathrm{H} »)$. 


\section{TABLeAU 5}

Effets de la nature des matières grasses sur les coefficients d'allométrie des différents constituants chimiques du corps par rapport au poids vif vide chez l'agneau mâle préruminant de race limousine entre la naissance et 3 semaines;

a) constituants du corps vide; b) constituants de la carcasse.

Effect of the type of lipid on the allometry coefficients of the different body chemical components relative to empty body weight in preruminant male Limousin lambs between birth and 3 weeks of age; a) empty body components; b) carcass components.

\begin{tabular}{|c|c|c|c|c|c|}
\hline \multirow{2}{*}{$\begin{array}{l}\text { Constituants } \\
\text { Components }\end{array}$} & \multirow{2}{*}{$\begin{array}{c}\text { Nature des } \\
\text { matières } \\
\text { grasses } \\
\text { Type } \\
\text { of lipid } \\
\end{array}$} & \multicolumn{2}{|c|}{$\begin{array}{c}\text { Constituants } \\
\text { du corps vide } \\
\text { Empty body components }\end{array}$} & \multicolumn{2}{|c|}{$\begin{array}{c}\text { Constituants } \\
\text { de la carcasse } \\
\text { Carcass components } \\
\end{array}$} \\
\hline & & $\mathbf{b} \pm \mathbf{S b} *$ & & $\mathbf{b} \pm \mathbf{S b} *$ & \\
\hline $\begin{array}{l}\text { 1. Données rapportées } \\
\text { au poids vif vide } \\
\text { Data relative to } \\
\text { empty body weight }\end{array}$ & & & & & \\
\hline$\underset{\text { Water }}{\text { Eau }} \cdots \cdots$ & $\begin{array}{l}\text { SC } \\
\text { SCT }\end{array}$ & $\begin{array}{l}0,826 \pm 0,033 \\
0,852 \pm 0,023\end{array}$ & $\mathrm{P}<0,01$ & $\begin{array}{l}1,141 \pm 0,038 \\
1,180 \pm 0,054\end{array}$ & $\mathbf{P}<0,01$ \\
\hline $\begin{array}{l}\text { Matière organique non } \\
\text { prot. } \ldots \ldots \ldots \ldots \ldots \ldots \\
\text { Non protein organic } \\
\text { mat. }\end{array}$ & $\begin{array}{l}\text { SC } \\
\text { SCT }\end{array}$ & $\begin{array}{l}2,545 \pm 0,252 \\
2,253 \pm 0,178\end{array}$ & $\mathrm{P}<0,001$ & $\begin{array}{l}2,232 \pm 0,378 \\
2,011 \pm 0,321\end{array}$ & $\mathbf{P}<0,05$ \\
\hline $\begin{array}{l}\text { Protéines } \\
\text { Protein }\end{array}$ & $\begin{array}{l}\text { SC } \\
\text { SCT }\end{array}$ & $\begin{array}{l}1,226 \pm 0,067 \\
1,205 \pm 0,096\end{array}$ & NS & $\begin{array}{l}1,670 \pm 0,184 \\
1,612 \pm 0,122\end{array}$ & NS \\
\hline $\begin{array}{l}\text { Cendres } \ldots \ldots \ldots \ldots \\
\text { Ashes }\end{array}$ & $\begin{array}{l}\text { SC } \\
\text { SCT }\end{array}$ & $\begin{array}{l}0,892 \pm 0,095 \\
0,860 \pm 0,084\end{array}$ & NS & $\begin{array}{l}0,901 \pm 0,086 \\
0,912 \pm 0,085\end{array}$ & NS \\
\hline $\begin{array}{l}\text { Energie } \ldots \ldots \ldots \ldots \\
\text { Energy }\end{array}$ & $\begin{array}{l}\mathrm{SC} \\
\mathrm{SCT}\end{array}$ & $\begin{array}{l}1,773 \pm 0,166 \\
1,615 \pm 0,091\end{array}$ & $\mathrm{P}<0,001$ & $\begin{array}{l}2,019 \pm 0,174 \\
1,861 \pm 0,233\end{array}$ & $\mathrm{P}<0,025$ \\
\hline $\begin{array}{l}\text { 2. Données rapporiées } \\
\text { à la masse délipidée } \\
\text { Data relative to } \\
\text { lean body mass }\end{array}$ & & & & & \\
\hline$\underset{\text { Water }}{\text { Eau }} \ldots \ldots \ldots \ldots \cdots$ & $\begin{array}{l}\text { SC } \\
\text { SCT }\end{array}$ & $\begin{array}{l}0,915 \pm 0,025 \\
0,932 \pm 0,015\end{array}$ & $\mathrm{P}<0,01$ & $\begin{array}{l}1,248 \pm 0,080 \\
1,285 \pm 0,062\end{array}$ & NS \\
\hline $\begin{array}{l}\text { Protéines } \\
\text { Proteins }\end{array}$ & $\begin{array}{l}\text { SC } \\
\text { SCT }\end{array}$ & $\begin{array}{l}1,360 \pm 0,071 \\
1,334 \pm 0,067\end{array}$ & NS & $\begin{array}{l}1,850 \pm 0,138 \\
1,759 \pm 0,103\end{array}$ & $P<0,025$. \\
\hline
\end{tabular}

* Coefficient d'allométrie et écart type - Allometry coefficient and standard error of mean (SEM). 
Il en est de même dans le corps entier des agneaux (-14,5 et $-7,8$ p. 100 respectivement pour les laits $« \mathrm{M} » ; 12,0$ et 4,3 p. 100 respectivement pour les laits « $\mathrm{H} »)$. L'allométrie de fixation de protéines ou de matières minérales dans la carcasse n'est pas modifiée.

Enfin, les allométries de la fixation d'eau ou de protéines rapportées à la «masse délipidée» (poids du corps entier vide diminué du poids de matière organique non protéique) révèlent (tabl. 5) que l'utilisation d'acide caproïque conduit à un accroissement de 2,5 p. 100 environ de la vitesse relative de fixation d'eau (significatif seulement dans le cas du corps entier, vide) et une diminution de 2 à 5 p. 100 de la vitesse relative de fixation de protéines (significative seulement dans le cas de la carcasse).

\section{Discussion}

\section{A. Appétibilité des aliments}

Comme les effets de l'huile de coprah sur l'appétibilité du lait (Theriez et al., 1973), ceux de la tricaproïne (lait "SCT 1H») ne se manifestent qu'à partir de la troisième semaine (fig. 1). Le fonctionnement de l'organisme ou le développement de la caillette à la naissance semblent donc insuffisants pour permettre une augmentation de l'ingestion de lait et de la vitesse de croissance du jeune agneau.

De plus, l'augmentation des consommations induite par la présence d'acide caproïque dans le lait se manifeste seulement au cours de l'essai 1 et n'est pas retrouvée dans les essais 2, 3 et 4 .

L'alimentation des agneaux en groupes, au sol, à différents stades de leur vie encadrant les périodes de mesures semble nécessaire à l'obtention des niveaux de consommation élevés et à l'extériorisation des différences d'appétibilité des laits.

\section{B. Efficacité de l'utilisation des aliments pour la croissance de l'agneau}

L'absence d'effets très nets de l'augmentation de la teneur en protéines des laits (de 24 à 31 p. 100 de la MS) sur l'efficacité de leur utilisation pour la croissance est en contradiction avec les observations de Chrou \& Jordan (1973) et celles de Walker \& Faichney (1964), mais en accord avec la plupart des travaux (Walker \& Cook, 1967 ; OWen \& Davies, 1970 ; Black, Pearce \& Tribe, 1973 ; Dove, Pearce \& Tribe, 1977). Cependant, l'analyse de variance indique un effet significatif pour l'ensemble des résultats. Par ailleurs, la supplémentation des laits « $\mathrm{SCM}$ » et «SCT $\mathrm{M}$ » en acides aminés indispensables peut améliorer l'efficacité de leur utilisation pour la croissance (WAlker \& Kirk, 1975 ; Dove, Pearce \& TRIBE, 1977) et masquer les effets éventuels des différences de teneurs en protéines.

En revanche, les effets de l'ingestion d'acide caproïque sont très importants. Le calcul montre que l'amélioration de la digestibilité des constituants du lait (AurousSeau, Vermorel \& Theriez, 1983) ne peut expliquer, au maximum, que la moitié des effets favorables de ce composé sur l'efficacité d'utilisation des aliments pour 
la croissance et que, en conséquence, il exerce aussi une action au niveau du métabolisme. Ces observations sont conformes à celles faites chez le veau (AurousSEAU, Vermorel \& Bouvier, 1983). Elles sont également en accord avec l'accroissement de la vitesse de croissance observée lorsqu'une partie du suif du lait est remplacé par de l'huile de coprah (Theriez et al., 1963, chez l'agneau; Roy et al., 1973, chez le veau). Le tiers des matières grasses des aliments utilisés dans la présente expérience étant apportées par de l'huile de coprah, les résultats obtenus confirment donc l'additivité des effets de l'acide caproïque et de ceux de l'huile de coprah (Aurousseau, Vermorel \& Bouvier, 1983, chez le veau préruminant). De plus, en dépit de la stimulation de la fixation de protéines corporelles par la supplémentation des laits en acides aminés indispensables (méthionine et lysine), l'acide caproïque exerce un effet additionnel sur la vitesse de croissance des agneaux et sur leur efficacité alimentaire.

\section{Composition corporelle des agneaux: influence de la concentration azotée et de la supplémentation en acides aminés}

Dans la présente expérience, les conséquences de l'augmentation de la teneur en protéines des aliments d'allaitement sur la composition corporelle sont limitées. Le rendement commercial ou les proportions de muscles dans la carcasse, la composition des tissus et la vitesse relative de fixation des différents tissus ou constituants chimiques ne sont pas modifiés contrairement aux observations de JAGUSCH, NortoN \& Walker (1970) et de Norton, Jagusch \& Walker (1970).

Ces résultats contradictoires semblent être liés à la supplémentation des laits en méthionine et en lysine qui permet d'obtenir une meilleure utilisation des protéines dans le cas des laits les moins riches (SCM et SCT M), d'après les travaux de Walker (1975), Walker \& Kirk (1975), Dove, Pearce \& Tribe (1977). En effet, comparés aux résultats obtenus antérieurement, avec des agneaux du même âge, Mérinos d'Arles (Benevent, 1971) ou New Zealand Romney (Broad \& Davies, 1980,1981 ), les coefficients d'allométrie du développement des tissus des agneaux Limousins employés dans la présente étude, sont plus élevés dans le cas du squelette ( 1,14 contre 0,69 ou 0,63 respectivement) et du muscle $(1,23$ contre 1,14 ou 0,93 respectivement). Ces différences ne sont pas liées au génotype des animaux puisque nous observons des valeurs plus faibles, voisines de celles décrites par BENEVENT ou par BROAD \& DAVIES chez des agneaux Limousins recevant un lait comportant 24 p. 100 de protéines, mais non supplémenté en méthionine et en lysine (résultats non publiés) : $0,79 \pm 0,07$ ou $1,01 \pm 0,05$ respectivement dans le cas du squelette ou du muscle.

L'examen de la composition chimique du corps entier des animaux aboutit aux mêmes conclusions. Comparées à celles décrites par RoBELIN et al. (1977) dans le cas d'agneaux mâles Limousins $\times$ Romanov $\times$ Ile-de-France à 1 et 5 semaines ou à celles observées par Searle, Graham \& O'Callaghan (1972), les valeurs rapportées ci-dessus correspondent à des teneurs en matière organique non protéique (lipides essentiellement), en cendres ou en énergie du même ordre mais révèlent des teneurs en eau plus faibles $(680 \mathrm{~g} / \mathrm{kg}$ contre $700 \mathrm{~g} / \mathrm{kg})$ et des teneurs en protéines plus élevées $(190 \mathrm{~g} / \mathrm{kg}$ contre $165 \mathrm{~g} / \mathrm{kg})$. Ces différences correspondent à des différences de composition du gain, soit $608 \mathrm{~g} / \mathrm{kg}$ contre $645 \mathrm{~g} / \mathrm{kg}$ pour l'eau et $207 \mathrm{~g} / \mathrm{kg}$ 
contre $170 \mathrm{~g} / \mathrm{kg}$ pour les protéines. Les valeurs rapportées dans la présente étude sont du même ordre de grandeur que celles obtenues par Jagusch, Norton \& Walker (1970) ou par Norton, Jagusch \& Walker (1970) dans le cas d'agneaux qui ont reçu des laits très riches en matières azotées ( 45 p. 100 de la matière sèche). Elles semblent donc bien s'expliquer par un effet favorable de la supplémentation des laits en méthionine et en lysine sur l'accrétion protéique comme il a été discuté plus haut.

L'augmentation relative du contenu en protéines du corps vide des agneaux utilisés dans la présente étude est également bien supérieure $(1,23$ contre 0,98$)$ à celle décrite par Robelin et al. (1977). Il en est de même pour les vitesses relatives de fixation de protéines dans la carcasse $(1,60)$ bien supérieures aux coefficients d'allométrie des protéines musculaires décrits par BroAd \& DAvIES, $1981(1,12)$. Comme précédemment, ces différences semblent être effectivement liées à la supplémentation des laits en méthionine et en lysine et non à une estimation erronée de la composition des agneaux à la naissance, puisque chez des agneaux de même génotype ingérant un lait comportant 24 p. 100 de MS sous forme de protéines non supplémentées en acides aminés (résultats non publiés) nous observons un coefficient d'allométrie de fixation de protéines dans la carcasse de 1,23 seulement. Ces modifications de la composition chimique corporelle des agneaux pourraient donc expliquer l'absence d'effets sur leur croissance observée par Theriez, Patureau-Mirand \& MolenAt (1977).

\section{Composition corporelle des agneaux.} Influence de l'ingestion d'acide caproïque

L'absence d'effet de l'ingestion d'acide caproïque sur la teneur en protéines du corps des agneaux ou des tissus déposés ainsi que sur l'allométrie de fixation de protéines corporelles différencie les effets de l'utilisation de ce composé de ceux de la supplémentation des laits en méthionine et en lysine.

Toutes choses égales par ailleurs, et en dépit de l'absence d'effets de l'acide caproïque sur la vitesse relative de développement des différents tissus, et en particulier des tissus adipeux, la présence de ce composé dans les aliments diminue la teneur de la carcasse ou du corps entier des animaux en matière organique non protéique (lipides essentiellement). Cette réduction est opérée au profit de leur teneur en eau, conformément aux lois du développement corporel (Tulloh, 1963 ; Lohman, 1971 ; Robelin, Geay \& Beranger, 1979).

Les données concernant l'utilisation de l'énergie métabolisable et de l'azote digestible ingérés pour la fixation dans les tissus feront l'objet d'une communication ultérieure, mais les résultats rapportés ci-dessus (augmentation de l'efficacité alimentaire sans modification de la teneur en protéines des tissus déposés, réduction des quantités des matières organiques non protéiques déposées, constituées principalement de lipides) sont en accord avec cet1x obtenus chez le veau préruminant (Aurousseau, VERMOREL \& BOUVIER, 1983) et montrent une stimulation de la fixation d'azote aux dépens de la fixation de lipides. Ces effets de l'acide caproïque sont obtenus en addition de ceux de l'huile de coprah présente dans les aliments témoin et de leur supplémentation en méthionine et en lysine. Mais cet acide augmente la fixation relative d'eau et diminue celle de protéines (tabl. 5), alors que nos données, comparées à celles obtenues par d'autres auteurs en absence de suppléments d'acides aminés indiquent que la supplémentation en méthionine et en lysine aurait un effet inverse. 
Ce dernier point mériterait d'être contrôlé et approfondi. En tout état de cause, les acides aminés ajoutés dans le lait interviennent principalement en tant que substrats tandis que les apports directs d'énergie facilement métabolisable sous forme d'acide caproïque permettent une couverture des besoins en liaisons riches en énergie plus efficace.

\section{Conclusion}

Les résultats de la présente expérience prouvent que l'intérêt de l'utilisation des acides gras à chaîne moyenne et plus particulièrement de l'acide caproïque pour l'alimentation du jeune préruminant ne se limite pas à l'amélioration de la digestibilité de l'énergie et de l'azote ingérés.

Ils montrent, en effet, une influence favorable sur la vitesse de croissance de l'agneau préruminant et sur l'efficacité d'utilisation des aliments pour la fixation de tissus qui dépasse le niveau permis par la meilleure digestibilité des laits enrichis en acide caproïque.

L'augmentation du gain de poids des animaux sans modification de l'allométrie de fixation des protéines corporelles et donc de la teneur en protéines des tissus déposés, mais accompagnée d'une diminution des quantités de matière organique non protéique fixées (lipides essentiellement) montre bien que l'utilisation d'acide caproïque améliore l'utilisation des acides aminés ingérés pour la fixation de protéines tissulaires aux dépens d'une diminution des quantités d'énergie utilisées pour la fixation de lipides.

Les relations entre les quantités de nutriments effectivement métabolisés dans l'organisme et la fixation de tissus nouveaux seront présentées dans une prochaine communication.

\section{Summary}

Combined effects of caproic acid and protein level in the milk replacers offered to preruminant lambs.

\section{II. - Growth and body composition of the lambs}

Growth rate, feed efficiency, body tissues and chemical composition in preruminant male lambs as affected by the type of lipid used (tallow-coconut oil "SC , or tallowcoconut oil-tricaproin, "SCT»), protein level (medium, «M», i.e. 24 p. 100 of dry matter, $\mathrm{DM}$, or high, «H», i.e. 31 p. 100 of $\mathrm{DM}$ ) or amount of tricaproin (4.5 or 11.4 p. 100 of DM) were studied in 4 trials (table 1). Growth and feed efficiency were compared between 3 and 21 days of age in 56 lambs from the Limousin breed (trials 1,2 and 4) and 16 lambs from the Ile-de-France breed (trial 3). Body tissues and chemical composition were studied in 24 lambs of the Limousin breed slaughtered at the age of 3 weeks (trial 2).

Daily growth rate of Limousin lambs increased $(\mathrm{P}<0,05)$ in "SCT-H » as compared to «SC-M》groups from about $206 \mathrm{~g} / \mathrm{d}$ up to $263 \mathrm{~g} / \mathrm{d}$ and was intermediate for the animals of the other groups "SC-H» and "SCT-M». In Ile-de-France lambs, the 
growth rate also increased $(+14$ p. $100, \mathrm{NS})$ in "SCT " as compared to " $\mathrm{SC}$ ». Feed efficiency was not altered by the increase in the protein level of the milk replacers, but significantly increased $(\mathrm{P}<0.05)$ by the caproic acid intake from about $939 \mathrm{~g} \mathrm{gain} / \mathrm{kg}$ DM intake or 40.6 gain/MJ gross energy in « SC 》 to about $1084 \mathrm{~g} \mathrm{gain} / \mathrm{kg} \mathrm{DM}$ or $48.8 \mathrm{~g}$ gain/MJ gross energy in the «SCT» groups (table 2).

The relative weights of the whole digestive tract and of the skin head and legs were higher in $" \mathrm{H}$ » as compared to " $\mathrm{M}$ » groups (table 3), but they were not modified by any of the experimental factors, type of lipid or protein level in the milk. The allometry coefficients for non carcass tissues, carcass, bones, muscles and adipose tissues were 0.801 , $1.275,1.232,1.144$ and 1.452 , respectively and were not altered by caproic acid intake, but they were higher than usual, for bones and muscles, which might be due to the methionine and lysine supplements.

Empty body composition or gain composition (table 5) showing high protein contents (189 $\mathrm{g} / \mathrm{kg}$ empty body or $206 \mathrm{~g} / \mathrm{kg}$ gain) is in favour of the effect of amino acid supplements on protein accretion. Caproic acid intake induced an increase in the allometry coefficient of water holding to the expense of non protein organic matter (table 5) so that water content in the empty body of the lambs increased and non protein organic matter decreased in the "SCT» groups (table 4).

Key words : caproic acid, growth, body composition, preruminant lamb.

Reçu en septembre 1983.

Accepté en janvier 1984.

\section{Références bibliographiques}

Aurousseau B., Theriez M., Daniel M., 1973. Influence de la nature des matières grasses incorporées dans l'aliment d'allaitement sur le métabolisme lipidique de l'agneau de boucherie. Ann. Biol. Anim. Biochim. Biophys., 13, 93-105.

Aurousseau B., Vermorel M., Bouvier J.C., 1983. Influence du remplacement d'une partie du suif d'un aliment d'allaitement par de la tricaproïne ou de l'huile de coprah, sur l'utilisation de l'énergie et de l'azote par le veau préruminant; influence du niveau d'alimentation antérieur. Reprod. Nutr. Dév., 23, 587-597.

Aurousseau B., Vermorel M., Theriez M., 1983. Effets combinés de lacide caproïque et de la concentration en protéines sur l'utilisation de laits artificiels par l'agneau préruminant. 1. Utilisation digestive des principaux constituants du régime. Ann. Zootech., 32, 441-458.

Benevent M., 1971. Croissance relative pondérale postnatale, dans les deux sexes des principaux tissus et organes de l'agneau Mérinos d'Arles. Ann. Biol. Anim. Biochim. Biophys., 11, 5-39.

Black J.L., Grifitiths D.A., 1975. Effects of liveweight and energy intake on nitrogen balance and total $\mathrm{N}$ requirements of lambs. Brit. J. Nutr., 33, 399-413.

Black J.L., Pearce G.R., Tribe D.E., 1973. Protein requirements of growing lambs. Br. J. Nutr., 30, 45-60.

Broad T.E., Davils A.S., 1980. Pre and post-natal study of the carcass growth of sheep. 1. Growth of dissectable lat and its chemical components. Anim. Prod., 31, 63-71.

Broad T.E., Davies A.S., 1981. Pre and post-natal study of the carcass growth of sheep. 3. Growth of dissectable and chemical components of muscle, and changes in the muscle : bone ratio. Anim. Prod., 32, 235-243.

ChIou P.W.S., JoRdan R.M., 1973. Ewe milk replacer diets for young lambs. III. Effect of age of lambs and dietary protein and fat levels on the body composition of young lambs. J. Anim. Sci., 36, 607-612.

Dove H., Pearce G.R., Tribe D.E., 1977. Utilization of amino acids by preruminant lambs. I. The effect of alteration in total essential amino acid intake at constant nitrogen intake. Aust. J. Agric. Res., 28, 917-932. 
Jagusch K.T., Norton B.W., Walker D.M., 1970. Body composition studies with the milk fed lambs. II. The effect of the age of the lambs and the proteins content of the diet on the chemical composition. J. Agric. Sci. Camb., 75, 279-285.

Lohman T.G., 1971. Biological variation in body composition. J. Anim. Sci., 32, 647-653.

Norton B.W., JAgusch K.T., Walker D.M., 1970. Body composition studies with the milk-fed lamb. III. The effect of the protein and energy intake on the composition of the live-weight gain. J. Agric. Sci. Camb., 75, 287-292.

OWEN J.B., Davies D.A.R., 1970. Milk replacers in the artificial rearing of lambs. J. Sci. Fd. Agric., 21, 340-342.

Robelin J., Geay Y., Beranger C., 1979. Evolution de la composition corporelle de jeunes bovins mâles entiers de race limousine entre 9 et 19 mois. II. Composition chimique et valeur calorifique. Ann. Zootech., 28, 191-208.

Robelin J., Theriez M., Arnal M., Ferrara M., 1977. Evolution de la composition chimique de jeunes agneaux mâles jusqu'à l'âge de 16 semaines. Ann. Zootech., 26, 69-81.

Roy J.H.B., Stobo I.J.F., Gaston H.J., Chotton S.M., Ganderton P., 1973. The nutrition of the veal calf. 5. Comparison of two margarine fats. Anim. Prod., 17, 97-107.

Searle T.W., Graham N.M.C., O'Callaghan M., 1972. Growth in sheeps. I. The chemical composition of the body. J. Agric. Sci., 79, 371-382.

Snedecor G.W., Cocheran W.G., 1971. Méthodes statistiques. Trad. H. Boelle et E. CAMaJI, Association de coordination technique agricole, Paris, Ed., 524-559.

Stokes G.B., Walker D.M., 1970. The nutritive value of fat in the diet of the milk fed lambs. 2. The effect of different dietary fats on the composition of the body fats. Br. J. Nutr., 24, 435-440.

Theriez M., Molenat G., Daniel M., Aurousseau B., 1973. L'allaitement artificiel des agneaux. III. Influence de la nature des matières grasses incorporées dans l'aliment d'allaitement. Ann. Zootech., 22, 185-197.

Theriez M., Patureau-Mirand P., Molenat G., 1977. Allaitement artificiel de l'agneau. IV. Comparaison de différentes teneurs en matières azotées dans le lait de remplacement. Ann. Zootech., 26, 297-313.

TULloH N.M., 1963. The carcass composition of sheep, cattle and pigs as function of body weight. In : Carcass composition and appraisal of meat animal, Ed. D.E. Tribe, East Melbourne, C.S.I.R.O., 5, 1-16.

Villette Y., Aurousseau B., 1981. Influence du poids à la naissance et du génotype sur la composition chimique de l'agneau nouveau-né. Ann. Zootech., 30, 285-298.

Walker D.M., Cook L.J., 1967. Nitrogen balance studies with the milk-fed lamb. 4. Effect of different nitrogen and sulphur intakes on live weight gain and wool growth and on nitrogen and sulphur balances. Br. J. Nutr., 21, 237-256.

WaLKER D.M., 1975. Methionine supplementation of milk proteins for preruminant lambs. II. Effect on wool growth, liveweight gain and body composition. Aust. J. Agric. Res., 26, 681-688.

Walker D.M., Faichney G.J., 1964. Nitrogen balance studies with the milk-fed lamb. 3. Effect of different nitrogen intakes on growth and nitrogen balance. Br. J. Nutr., 18, 295-306.

WALKER D.M., KirK R.D., 1975. Methionine supplementation of milk proteins for preruminant lambs. I. Effect of protein concentration and source of carbohydrate on nitrogen balance. Aust. J. Agric. Res., 26, 673-679.

Welch J.G., Vander Noot G.W., Gilbreath R.L., 1963. Effect of feeding milk replacers with varying amounts of fat for hothouse lamb production. I. Anim. Sci., 22, $155-158$. 


\section{AnNeXe 1}

Composition corporelle de l'agneau limousin à l'âge de 3 semaines (essai 2) : moyennes et écarts types.

Body composition of 3 weeks old Limousin lamb (trial 2) : mean and SEM.

\begin{tabular}{|c|c|c|c|c|}
\hline \multirow{2}{*}{$\begin{array}{l}\begin{array}{l}\text { Nature des matières grasses } \\
\text { Type of lipid }\end{array} \\
\text { Concentration en protéines } \\
(\% \text { MS) .................. } \\
\text { Protein concentration }(\% \text { DM })\end{array}$} & \multicolumn{2}{|c|}{$\begin{array}{c}\text { Suif-coprah (SC) } \\
\text { Tallow-coconut oil }(S C)\end{array}$} & \multicolumn{2}{|c|}{$\begin{array}{l}\text { Suif-coprah-tricaproïne } \\
\text { (SCT) } \\
\text { Tallow-Coconut oil- } \\
\text { tricaproin (SCT) } \\
(S C T)\end{array}$} \\
\hline & 24 & 30 & 24 & 30 \\
\hline $\begin{array}{l}\text { Aliment d'allaitement } \ldots \ldots \ldots \ldots \\
\text { Milk replacer }\end{array}$ & SC $2 \mathrm{M}$ & $\mathrm{SC} 2 \mathrm{H}$ & SCT $2 \mathrm{M}$ & $\mathrm{SCT} 2 \mathrm{H}$ \\
\hline $\begin{array}{l}\text { Poids de naissance }(\mathrm{kg}) \ldots \ldots \\
\text { Birth weight }(\mathrm{kg})\end{array}$ & $3,3 \pm 0,3$ & $3,5 \pm 0,3$ & $3,3 \pm 0,4$ & $3,3 \pm 0,4$ \\
\hline $\begin{array}{l}\text { Age à l'abattage (jours) } \ldots \ldots \\
\text { Age at slaughter (days) }\end{array}$ & $23,3 \pm 0,9$ & $23,3 \pm 1,0$ & $23,7 \pm 1,1$ & $23,3 \pm 1,0$ \\
\hline $\begin{array}{l}\text { Poids à l'abattage }(\mathrm{kg}) \\
\text { Weight at slaughter }(\mathrm{kg})\end{array}$ & $7,6 \pm 0,8$ & $8,3 \pm 0,8$ & $7,7 \pm 0,7$ & $8,4 \pm 1,3$ \\
\hline $\begin{array}{l}\text { Sang recueilli }(\mathrm{g}) \ldots \ldots \ldots \ldots \\
\text { Sampled blood }(g)\end{array}$ & $241 \pm 102$ & $332 \pm 58$ & $275 \pm 108$ & $333 \pm 72$ \\
\hline $\begin{array}{l}\text { Foie }(g) \ldots \ldots \ldots \ldots \ldots \ldots \ldots \\
\text { Liver }(g)\end{array}$ & $248 \pm 20$ & $229 \pm 37$ & $243 \pm 35$ & $249 \pm 34$ \\
\hline $\begin{array}{l}\text { Cour et poumons }(\mathrm{g}) \ldots \ldots \ldots \\
\text { Heart and lungs }(g)\end{array}$ & $580 \pm 89$ & $594 \pm 167$ & $556 \pm 67$ & $512 \pm 117$ \\
\hline $\begin{array}{l}\text { Tube digestif }(g) \ldots \ldots \ldots \ldots \ldots \\
\text { Gastrointestinal tract }(g)\end{array}$ & $493^{a} \pm 46$ & $615^{\mathrm{b}} \pm 54$ & $514^{a} \pm 64$ & $585^{b} \pm 29$ \\
\hline $\begin{array}{l}\text { Peau, tête et pattes }(\mathrm{g}) \ldots \ldots \\
\text { Skin, head and legs }(\mathrm{g})\end{array}$ & $1454^{a} \pm 140$ & $1627^{a b} \pm 180$ & $1490^{a} \pm 140$ & $1719^{b} \pm 284$ \\
\hline $\begin{array}{l}\text { Os de la carcasse }(\mathrm{g}) \ldots \ldots \ldots \ldots \\
\text { Carcass bones }(g)\end{array}$ & $964 \pm 92$ & $1066 \pm 110$ & $967 \pm 110$ & $1010 \pm 164$ \\
\hline $\begin{array}{l}\text { Muscles }(g) \ldots \ldots \ldots \ldots \ldots \ldots \\
\text { Muscle }(g)\end{array}$ & $2597 \pm 238$ & $2667 \pm 430$ & $2666 \pm 218$ & $2845 \pm 458$ \\
\hline $\begin{array}{l}\text { Tissus adipeux sous-cutanés }(\mathrm{g}) \\
\text { Subcutaneous adipose tissue }(\mathrm{g})\end{array}$ & $169 \pm 42$ & $149 \pm 48$ & $138 \pm 38$ & $157 \pm 50$ \\
\hline $\begin{array}{l}\text { Tissus adipeux internes }(\mathrm{g}) \ldots \\
\text { Internal adipose tissue }(\mathrm{g})\end{array}$ & $98 \pm 22$ & $107 \pm 24$ & $93 \pm 14$ & $105 \pm 16$ \\
\hline $\begin{array}{l}\text { Tissus adipeux intermuscul. (g) } \\
\text { Intermuscular adipose tissue }(g)\end{array}$ & $392 \pm 96$ & $410 \pm 120$ & $337 \pm 70$ & $461 \pm 124$ \\
\hline
\end{tabular}

